\title{
Modeling and Filter Design through Analysis of Conducted EMI in Switching Power Converters
}

\author{
R. Vimala ${ }^{\dagger}$, K. Baskaran*, and K. R. Aravind Britto** \\ ${ }^{\dagger}$ Dept. of EEE, PSNA College of Engineering and Technology, Dindigul, India \\ Dept. of CSE, Govt. College of Technology (GCT), Coimbatore, India \\ ${ }^{* *}$ Dept. of ECE, RVS College of Engineering and Technology, Dindigul, India
}

\begin{abstract}
This paper presents a filter analysis of conducted Electro-Magnetic Interference (EMI) in switching power converters (SPC) based on noise impedances. The EMI characteristics of SPC can be analytically deduced from a circuit theoretical viewpoint. The analytical noise model is investigated to get a full understanding of the EMI mechanism. It is shown that with suitable and justified model, filters pertinent to EMI noise is investigated. The EMI noise is identified by time domain measurements associated with an isolated half-bridge ac-dc converter. Practical filters like LC filter, $\pi$ filter and complete EMI filters are investigated. The proposed analysis and results can provide a guideline for improving the effectiveness of filtering schemes in SPC. Experimental results are also included to verify the validity of the proposed method. The results obtained satisfy the Federal Communications Commission (FCC) class A and class B regulations.
\end{abstract}

Key words: Common-Mode (CM), Differential-Mode (DM), Eelectro-Magnetic Compatibility (EMC), Conducted EMI, Switching Power Converters (SPC)

\section{INTRODUCTION}

EMI emissions are always a great concern due to the modern trends in power electronics such as fast switching with large amount of current at high voltages and high frequency in switching devices like Metal Oxide Semiconductor Field Effect Transistors (MOSFET), Insulated Gate Bipolar Transistors (IGBT) and Gate Turn Off Thyristors (GTO). The frequency range of the conducted emissions limit is from $450 \mathrm{kHz}$ to $30 \mathrm{MHz}$ for the FCC class A and class B regulations and $150 \mathrm{kHz}$ to $30 \mathrm{MHz}$ for the German Verband Deutscher Elektroniker (VDE) regulations. Radiated emissions are generally measured at much higher frequencies, namely beyond $30 \mathrm{MHz}$ up to several $\mathrm{GHz}$. Conducted EMI in power converters has become increasingly important due to its wide applications. Recently, EMI problems have become more serious for power electronic systems. The process of EMI mitigation normally involves

Manuscript received Mar. 30, 2011; revised May 18, 2012

Recommended for publication by Associate Editor Tae-Woong Kim.

${ }^{\dagger}$ Corresponding Author: vimala79@rediffmail.com

Tel: +91-9965497316, PSNA College of Engineering and Technology

*Dept. of Computer Science and Eng., Government College of

Technology (GCT), India

${ }^{* * *}$ Dept. of Electronics and Communication Eng., RVS College of

Engineering and Technology, India the designing of filters. The traditional cut-and-try approach has been abandoned by engineers due to its time-consuming and inefficient defects. Therefore, effective solutions for the analysis and suppression of conducted EMI noise have become great concerns.

Conventionally, conducted EMI noise is caused by two mechanisms: Differential Mode (DM) noise and Common Mode (CM) noise. Furthermore, EMI filter should be designed for CM noise and DM noise, respectively. Therefore, the first step in designing EMI filter is to separate the CM and DM noise. A two way $0^{\circ}$ combiner (ZFSC -2-6-75) and a two way $180^{\circ}$ power combiner (ZFSCJ-2-1) are used to measure the total DM and CM noise via the selection of a three way built-in switch. Moreover, in order to suppress conducted EMI noise, the proper design of EMI filter is necessary. In general, DM noise is related to switching current and CM noise is related to the capacitive coupling of the switching voltage with the Line Impedance Stabilizing Network (LISN), which is used in standard conducted EMI measurement.

Accurate modeling of EMI noise generation and propagation in power converters is the first step in predicting and managing the EMI noise in a system. Effective EMI prediction often relies on an engineer's experience or extensive numerical simulation models [1]-[3]. Owing to the effectiveness of 


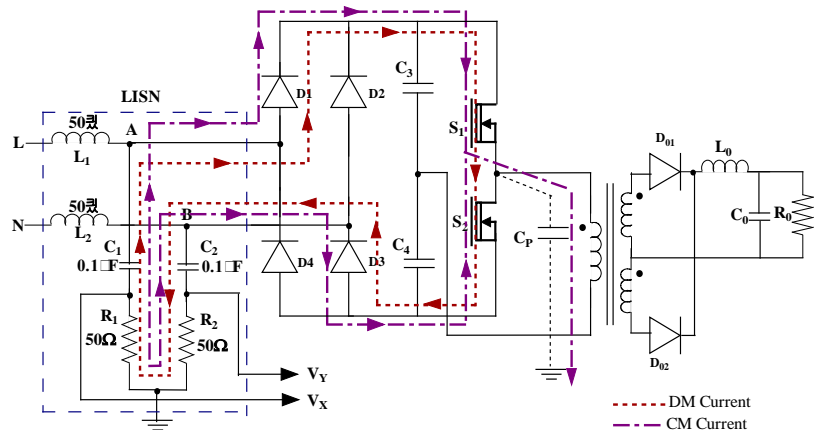

Fig. 1. Conducted EMI coupling path of Half-bridge isolated ac-dc converter

filtering conducted EMI noise separately by CM and DM noise, each mode of the noises is dealt with by the respective section of an EMI filter [4]. New techniques for designing EMI filters have been developed recently by use of a noise separator, which is used to separate DM and CM noise [5].

\section{MODEL DESCRIPTION}

\section{A. DM/CM Coupling}

Fig.1 shows the configuration of the conducted EMI measurement for an isolated half-bridge ac-dc converter. The power source is provided through a LISN, which is required by the conducted EMI measurement and contains inductors, capacitors, and resistors. In the LISN, there are two $50 \mu \mathrm{H}$ inductors $-\mathrm{L}_{1}$ and $\mathrm{L}_{2}$, two $0.1 \mu \mathrm{F}$ capacitors $-\mathrm{C}_{1}$ and $\mathrm{C}_{2}$ and two $50 \Omega$ resistors $-R_{1}$ and $R_{2}$. For noise frequency, the two inductors $L_{1}$ and $L_{2}$ present high impedance and two $0.1 \mu \mathrm{F}$ capacitors $\mathrm{C}_{1}$ and $\mathrm{C}_{2}$ present low impedance. The noise voltage coupled through the two resistors $R_{1}$ and $R_{2}$ is counted as conducted EMI. For power-line frequency, the inductors are essentially short, the capacitors are essentially open, and the power is passed through to supply the test system.

According to conventional theory, DM noise is defined as the voltage difference between the two LISN resistors, $V_{X}-V_{Y}$, while CM noise is defined as the average voltage of the two LISN resistors, $\left(\mathrm{V}_{\mathrm{X}}+\mathrm{V}_{\mathrm{Y}}\right) / 2$. For conducted EMI noise frequency, two $50 \mu \mathrm{H}$ inductors present high impedances and two $0.1 \mu \mathrm{F}$ capacitors present small impedances. The voltages measured across the two $50 \Omega$ impedances are defined by the conducted EMI, $\mathrm{V}_{\mathrm{X}}$ is the line-side EMI, and $\mathrm{V}_{\mathrm{Y}}$ is the neutral-side EMI. DM noise voltage and CM noise voltage are expressed in (1) and (2).

$$
\begin{array}{r}
\mathrm{V}_{\mathrm{CM}}=\left(\mathrm{V}_{\mathrm{X}}+\mathrm{V}_{\mathrm{Y}}\right) / 2 \\
\mathrm{~V}_{\mathrm{DM}}=\left(\mathrm{V}_{\mathrm{X}}-\mathrm{V}_{\mathrm{Y}}\right) / 2
\end{array}
$$

During normal operation, the dc link is clamped at a fixed voltage by the capacitances $\mathrm{C}_{3}$ and $\mathrm{C}_{4}$. When the ac side line voltage is larger than the capacitance voltage, the diode bridge is $\mathrm{ON}$, and when line voltage is smaller than the capacitance voltage, the diode bridge is OFF. DM noise current coupling path, as indicated in Fig.1, passes through the input rectifier diodes $\left(D_{1}\right.$ and $\left.D_{3}\right)$. The measured DM noise fluctuates with

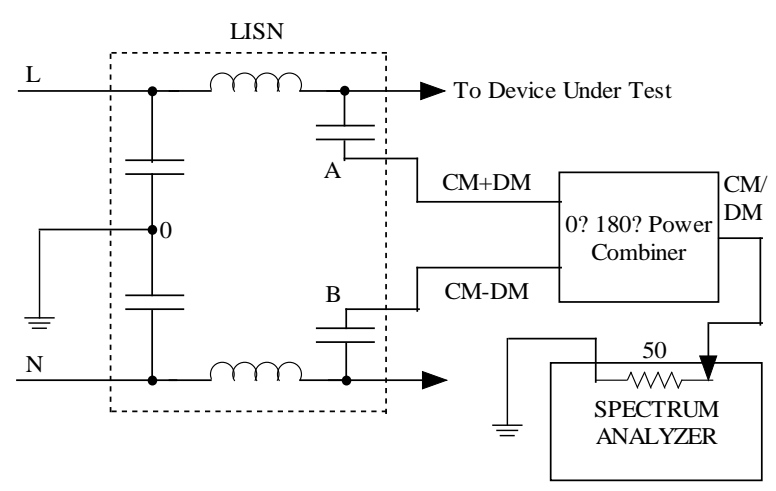

(a)

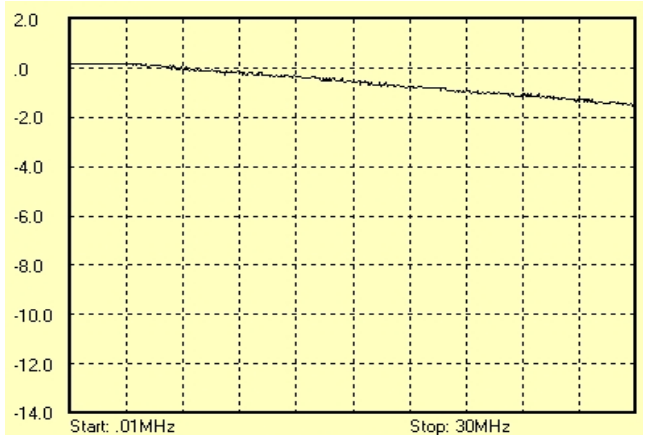

(b)

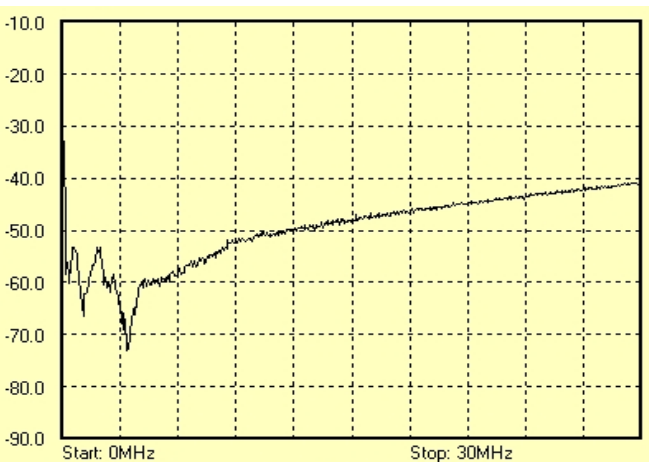

(c)

Fig. 2. (a) $0^{\circ} / 180^{\circ}$ Power Combiner. (b) CMIL of the noise separating network. (c) DMRR of the noise separating network.

time because the rectifier diodes are ON and OFF during half a supply cycle. However, CM noise is independent of the conduction state of the rectifier. The noise magnitude should be larger when the diodes are "ON" because the noise current couple $R_{1}$ and $R_{2}$ with less impedance in the path. The DM noise is higher when the rectifier is OFF than that when it is ON.

\section{B. EMI Noise Separator}

The noise separator is built using the principle of noise rejection accomplished by using power combiners. Fig.2 (a) shows diagram depicting the basic concept of a noise separator in which the two signals derived from the LISN, consist of both CM and DM noise. One of the signals is the 


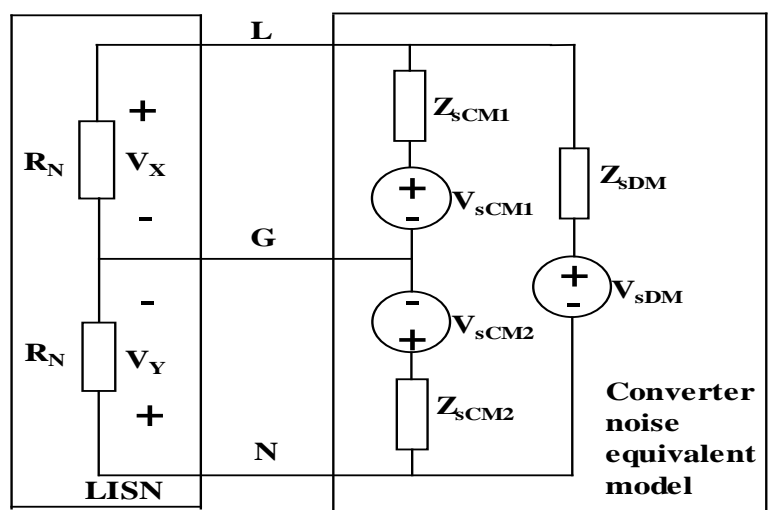

Fig. 3. Noise model for essential coupling path.

vector sum of the two modes of noise $(\mathrm{CM}+\mathrm{DM})$ and the other signal is the vector difference of the two modes of noise (CM-DM). The CM current is evenly divided between the two input terminals, which is often true except in extreme cases. There are four parameters that need to be defined to evaluate a noise separating network. These parameters are Common Mode Insertion Loss (CMIL), Differential Mode Insertion Loss (DMIL), Common Mode Rejection Ratio (CMRR) and Differential Mode Rejection Ratio (DMRR). The transmission coefficient of the noise separating network are defined in (3).

$$
\mathrm{S}_{21}=20 \log (\mathrm{V} 2 / \mathrm{V} 1)(\mathrm{dB})
$$

V1 and V2 are the input and output of the network. Transmission coefficient $S_{21}$ is CMIL/DMIL when V1 and V2 represent the same mode signals, and $S_{21}$ is CMRR/ DMRR when V1 and V2 represent different mode signals [6]. It should be mentioned that the insertion loss should not be more than $5 \mathrm{~dB}$ and the rejection ratio should not be less than $40 \mathrm{~dB}$. A spectrum analyzer and $0^{\circ} / 180^{\circ}$ combiners are used to measure the characterization parameters of the noise separating network [7]. The CMIL results of the high-performance noise separating network is shown in Fig.2 (b). As the frequency goes up, the CMIL declines slightly but remains above $-2 \mathrm{~dB}$. The DMRR result of the highperformance noise separating network is shown to have good performance in Fig.2(c). The DMRR rises with an increasing frequency, and at $30 \mathrm{MHz}$, the maximum frequency of conducted EMI noise measurement, the DMRR successful remains below $-40 \mathrm{~dB}$. The measurement results prove that the noise separating network separate the conducted EMI noise efficiently.

\section{Model Implementation}

Fig.3 shows an equivalent circuit model for a description of the essential coupling paths between the converter and the LISN's three physical terminals L, N, and G. Assume a linear model for the converter's physical circuit made up of a simple combination of three impedance elements together with three voltage sources. The overall noise is equal to the sum of all the components from the individual analysis of all the

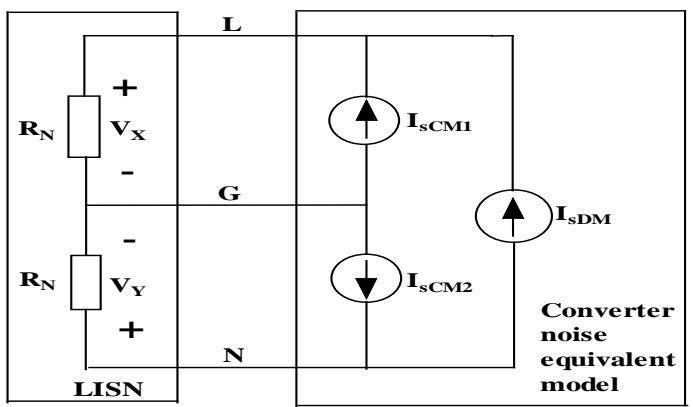

Fig. 4. Simplified noise model.

harmonics for a linear model. By dealing with EMI noise in this way, all of the details of the internal responses with the converter are lost, and only the external responses at the LISN input terminals are maintained [8]. The characteristics of the LISN are well defined. Each phase of the LISN is represented by $50 \Omega$ impedance.

$I_{\mathrm{SCM}}$ represents the SPC noise source current and $Z_{\mathrm{SCM}}$ represents the noise source impedance. $Z_{\mathrm{sDM}}$ is the $\mathrm{DM}$ noise impedance of the SPC. The linear model consists of one equivalent noise voltage source which is connected to the LISN through coupling impedance. EMI is coupled from three voltage sources through three impedances, i.e., $\mathrm{V}_{\mathrm{sDM}}$ and $\mathrm{Z}_{\mathrm{sDM}}$ are $\mathrm{DM}$ noise voltage source and impedance, while $\mathrm{V}_{\mathrm{SCM} 1}, \mathrm{~V}_{\mathrm{sCM} 2}, \mathrm{Z}_{\mathrm{sCM} 1}$, and $\mathrm{Z}_{\mathrm{SCM} 2}$ indicate $\mathrm{CM}$ noise voltage sources and impedances, respectively. The LISN is represented by two resistors $R_{N}$. These voltage sources depend on high $\mathrm{d} v / \mathrm{dt}$ and high di/dt slew rates as well as the circuit parasitic parameters, device package, and layout. Normally $Z_{\mathrm{sCM} 1}$ and $\mathrm{Z}_{\mathrm{sCM} 2}$ are high source impedances because they are associated with parasitic capacitance to the earth. Assume that $Z_{\mathrm{sCM} 1}>>50$ and $\mathrm{Z}_{\mathrm{sCM} 2}>>50$. Then obtain Fig 4.

The CM and DM currents are expressed in (4) - (6).

$$
\begin{gathered}
I_{S C M 1}=\frac{V_{S C M 1}}{Z_{S C M 1}} \\
I_{S C M 2}=\frac{V_{S C M 2}}{Z_{S C M 2}} \\
I_{S D M}=\frac{V_{S D M}}{Z_{S D M}}
\end{gathered}
$$

The effectiveness of an EMI filter depends on both the filter itself and the noise source impedance. For CM noise, the source is modeled by a current source in parallel with high source impedance $Z_{s}$. For DM noise, the source is modeled by a voltage source in series with low impedance or a current source in parallel with high impedance depending on the state of the input rectifier diodes. When two of the rectifier diodes are conducting, the noise source is modeled by a voltage source in series with a low impedance source $Z_{s}$. When all the four diodes are cut off, the noise is modeled by a current source in parallel with a high source impedance $Z_{p}$. Therefore, DM noise equivalent circuit fluctuates between these two models at two times the line frequency. $Z_{s}$ is 


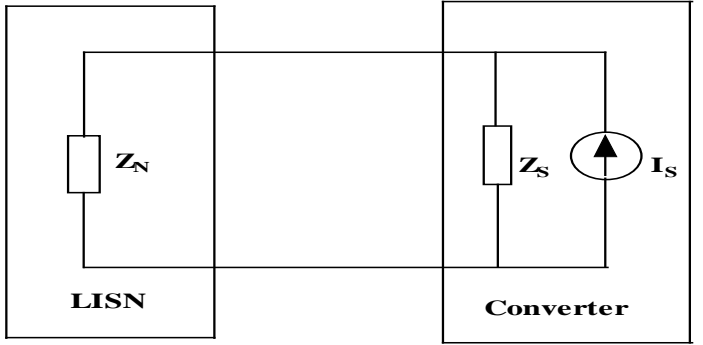

Fig. 5. Simplified EMI noise lumped circuit model.

associated with wire inductance and resistance, and $Z_{p}$ is associated with diode parasitic capacitance. These source impedances depend on parasitic parameters and are therefore package dependent. Although the source impedances is measured, this is particularly difficult. Two simplified equivalent circuits, one for CM emissions and another for DM emissions, are obtained.

\section{EMI Model for Symmetrical Circuit}

The model in Fig.3 contains all essential coupling paths and circuit parameters to provide a full picture of the EMI conduction and coupling mechanism. The DM voltage (with $\mathrm{R}_{\mathrm{N}}=50 \Omega$ ) is written in (7).

$V_{D M}=\frac{100 V_{s D M}}{Z_{S D M}+100}+\frac{50 Z_{s D M}}{Z_{s D M}+100}\left(I_{S C M 1}-I_{s C M 2}\right)$

From the equation (7), total DM noise has two parts: 1) the first part is determined by $\mathrm{DM}$ noise source $\mathrm{V}_{\mathrm{sDM}}$ and impedance $\mathrm{Z}_{\mathrm{sDM}}$, and is called Intrinsic Differential Mode (IDM) noise and 2) the second part is the difference of two CM current sources through the DM impedance and LISN, and is called Non-Intrinsic Differential Mode (NIDM) or Mixed Mode (MM) noise. It is clear that the MM noise is caused by an unbalanced CM current which flows through the two LISN branches. By following the above deductions, DM and CM noise source model is then be reduced to a simple two-port lumped circuit model, as shown in Fig. $5 . Z_{N}$ is $2 R_{N}$ in the case of $\mathrm{DM}$ and $0.5 \mathrm{R}_{\mathrm{N}}$ in the case of $\mathrm{CM}, \mathrm{Z}_{\mathrm{S}}$ is $\mathrm{Z}_{\mathrm{sDM}}$ in the case of DM and $0.5 Z_{\mathrm{SCM} 2}$ in the case of CM, and $\mathrm{I}_{\mathrm{S}}$ is $\mathrm{I}_{\mathrm{sDM}}$ in the case of DM and $\mathrm{I}_{\mathrm{SCM} 1}$ in the case of CM.

For reducing complexity, the EMI issues using very simple models always consider the combination of impedance $Z_{S}$ and source $V_{\mathrm{S}}$. These sources and impedances depend on parasitic parameters. Therefore, they are determined by converter package and layout. Although the sources and impedances are measured by a particular method [9], this is not in the scope of this paper. The major components of DM noise source impedance are the turned-on resistance of the rectifying diodes along with the Equivalent Series Resistance (ESR) and Equivalent Series Inductance (ESL) of the bulk capacitor. The major components of CM noise source impedance are the unintentional capacitance between the switching device and its heat-sink, parasitic capacitance between the heat-sink and the grounded chassis, the parasitic capacitances between the other devices or wires, which carry pulsating voltage waveform, and the grounded chassis.

To measure the impedances $\left(\mathrm{Z}_{\mathrm{sCM} 1}, \mathrm{Z}_{\mathrm{sCM} 2}\right.$, and $\left.\mathrm{Z}_{\mathrm{sDM}}\right)$ and voltage sources $\left(\mathrm{V}_{\mathrm{sCM} 1}, \mathrm{~V}_{\mathrm{sCM} 2}\right.$, and $\left.\mathrm{V}_{\mathrm{sDM}}\right)$ in Fig. 3, a piece of impedance element (in series or shunt) is inserted between the LISN and the converter. The noise voltage measured on the LISN changes, and the model parameter values are derived from a circuit theoretical viewpoint. For DM noise model identification, the shunt-insertion method is proposed because DM source impedance is small and $\left|Z_{\mathrm{sCM} 1}\right|<2 R_{N}$ is easily satisfied. However, for CM noise model identification, the series insertion method is proposed because CM source impedance is large and $\left|Z_{\mathrm{sDM}}\right|>\mathrm{R}_{\mathrm{N}}$ and $\left|\mathrm{Z}_{\mathrm{sCM} 2}\right|>\mathrm{R}_{\mathrm{N}}$ are easily satisfied [10]. Therefore, $Z_{\text {sCM1 }}$ is no more than several ten ohms, but $\mathrm{Z}_{\mathrm{sDM}}$ and $\mathrm{Z}_{\mathrm{sCM} 2}$ are always bigger than several hundred ohms in the frequency range of $150 \mathrm{kHz}-30 \mathrm{MHz}$.

The DM noise source is always considered as the pulse current of the power switch device. The CM noise source is associated with the high rate of the changing voltage put on the parasitic capacitances between the circuit and the ground. However, owing to the complexity of EMI noise coupling, the theoretical modeling of the parasitic elements has been a very challenging task. This is due to the fact that the parasitic elements are difficult to identify and the parasitic elements may be physically inaccessible inside the module package.

\section{ANALYSIS FILTERS FOR NOISE SUPPRESSION}

After the filter circuit has been selected, the component values in that circuit must be determined. The Y-capacitors are easily determined from the leakage current limit, which is given in the safety standards applicable to particular equipment. With known Y-capacitors, the CM inductance is calculated from (8).

$$
L=\frac{1}{4 \pi^{2} f_{c}^{2} C}
$$

where, $f c$ is the corner frequency, which is defined as the intersection between $0 \mathrm{~dB}$ line and a line with slope of 40 $\mathrm{dB} /$ decade that is tangent to the required CM insertion loss. Similarly, the DM corner frequency is determined from the required $\mathrm{DM}$ insertion loss. When the $\mathrm{CM}$ inductance is being calculated, the capacitance $\mathrm{C}$ in (8) should be twice the $C_{Y}$ value. The CM inductance consists of the CM choke's inductance and half of the inductance of the DM inductors, if they are decoupled inductors. If the DM inductors are coupled, they have negligible CM inductance and thus the $\mathrm{CM}$ inductor should have an inductance, equal to the required CM inductance, obtained by (8).

The selection of the filter components involves not just the calculation of suitable capacitance and inductance values, but also other considerations, such as the inductor core material, the dielectric of the capacitor, voltage and current ratings, the 


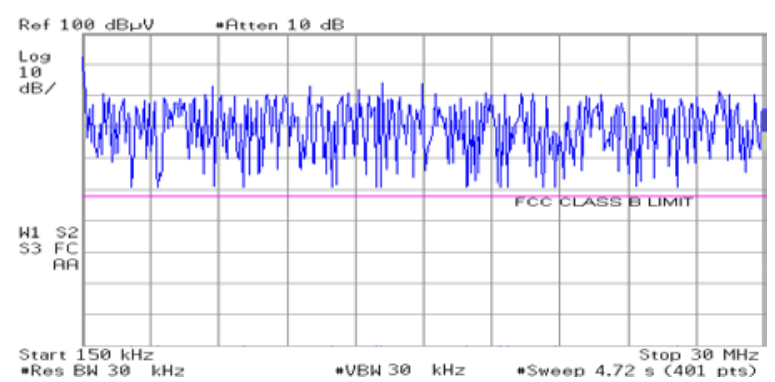

Fig. 6. Total noise spectrum without filter.

voltage drop at line frequency, size, weight, etc. $\mathrm{L}_{\mathrm{DM}}$ is a $\mathrm{DM}$ choke and $C_{X}$ is a DM capacitor (called an " $X$ " capacitor). When filtering DM noise, an X capacitor is used to shunt noise from one line to another through the low impedance of the capacitor, thus returning the noise to the EMI source. For DM noise, DM choke should have suppressing effect when the two paths are unbalanced. Even though current flow is unbalanced, the magnitude of each current is attenuated by the DM choke and therefore, the DM noise is suppressed.

To effectively suppress the DM noise, capacitor is always used together with the DM chokes. The measurement of the conducted EMI noise is obtained from spectrum analyzer Agilent E4411B (9 KHz - $1.5 \mathrm{GHz})$. After considering a measure bandwidth of $10 \mathrm{kHz}$ and normalized in $\mathrm{dB} \mu \mathrm{V}$ [11], the spectra of the total EMI without a filter is given in Fig. 6. The fluctuation of the EMI at a particular frequency is displayed by using the zero span mode of a spectrum analyzer. Using this mode, magnitude of selected noise frequency is displayed with respect to time. Another important issue is that the insertion of the filter should not affect the stability of the SPC and it affect the dynamics if the output impedance of the DM filter part is much less than the input impedance of the converter from dc to the gain crossover frequency of converter's control loop [12]-[15].

This requires that the DM filter should be well damped to avoid impedance peaking and consequent possible violation of this criterion. To effectively suppress DM noise, the balancing capacitor should provide a balanced impedance such that the currents flowing through the two LISN resistors R1 and R2 are the same, irrespective of $\mathrm{C}_{\mathrm{p}}$ 's charged condition. Under this condition, the choice of the value for $C_{x}$ depends on the frequency and the filter topology. The value of $C_{x}$ is calculated from (9).

$$
\mathrm{X}_{\mathrm{c}}=1 /\left(2 \pi \mathrm{fC}_{\mathrm{x}}\right) ; \mathrm{X}_{\mathrm{c}}<<50 \Omega
$$

$\mathrm{f}=$ starting frequency of EMI noise;

(150 kHz for VDE and $450 \mathrm{kHz}$ for FCC)

The filter attenuation is defined in (10). $\mathrm{V}_{\text {noise }}$ without filter is measured at the LISN output, when no EMI filter element is added. $\mathrm{V}_{\text {noise }}$ with filter is measured at the LISN output when a filter is added.

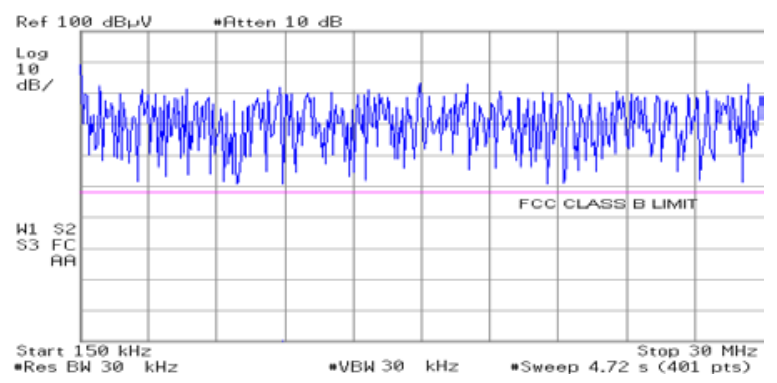

Fig. 7. CM noise spectrum without filter.

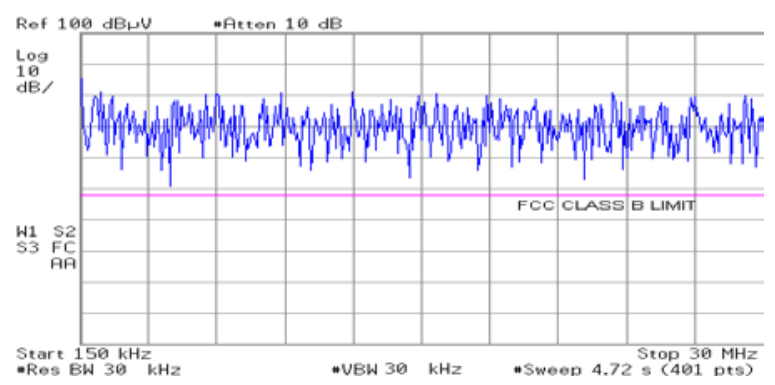

Fig. 8. DM noise spectrum without filter.

$$
\text { Afilter }=\frac{\text { Vnoise (without filter) }}{\text { Vnoise (with filter) }}
$$

The topology should be selected according to the source and load impedances. This approach considered filter topology only but did not mentioned the relationship of noise source impedance (Zs) and noise load impedance (LISN), which affects the EMI filter performance [16].

\section{EMI FILTER DESIGN PROCEDURE}

In this section, a method for designing an EMI filter using the maximum and minimum CM and DM noise impedances is introduced. An ac-dc half-bridge converter is used as an example. The objective is to design an EMI filter, so that the SPC will pass the FCC 15 Class B regulations for conducted EMI. The CM and DM filters are designed separately. The maximum or minimum worst case noise impedance is considered for each filter. After completion of the CM and DM filters, they are put together to make the complete filter [17]. The CM and DM noise spectra of an unfiltered ac-dc SPC, operating under full load, are illustrated in Fig.7 and Fig.8, respectively.

The procedure for designing the EMI filter is summarized as follows:

1) Separate the CM and DM noise spectrum of the SPC.

2) Measure the noise voltage, $V_{\text {noise }}$, with and without a simple filter (eg., a capacitor). Then use the attenuation to calculate the maximum CM and DM noise impedances for the frequency range under consideration $(0.15-30 \mathrm{MHz}$ for the FCC 15).

3) Design the EMI filter using the maximum or minimum magnitude of the noise impedance, which ever yields the least 


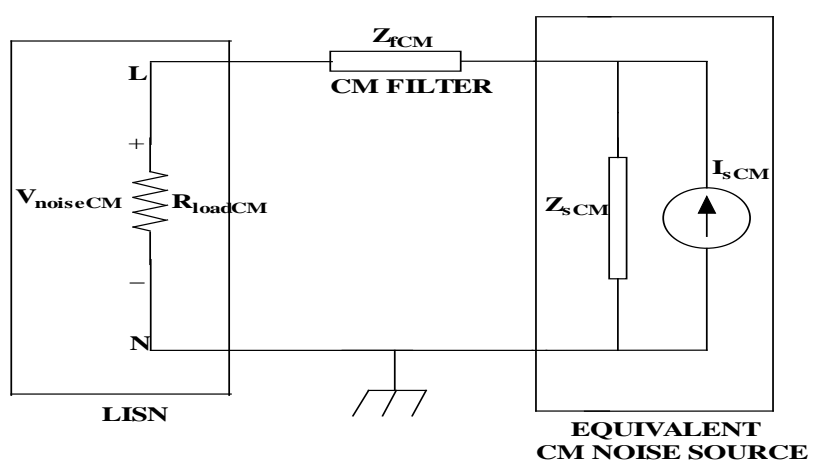

Fig. 9. CM Equivalent Circuit.

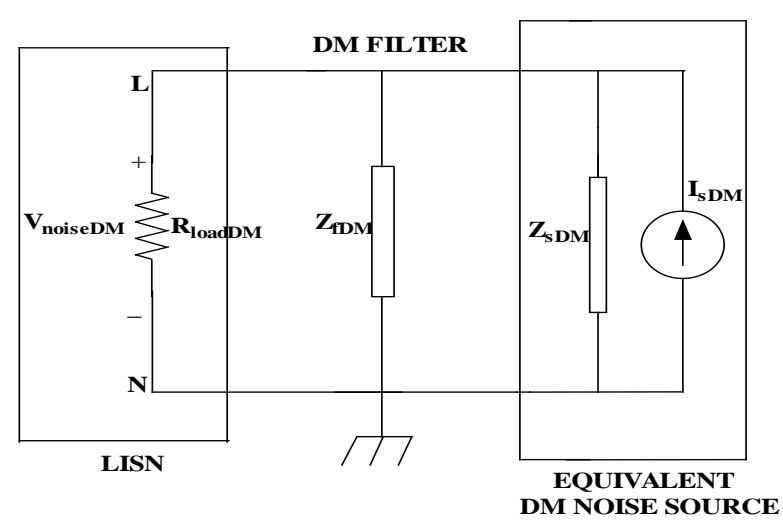

Fig. 10. DM Equivalent Circuit.

attenuation.

4) Test the completed EMI filter.

In order to measure the maximum and minimum possible value of CM noise source impedance, a test inductor is added at the input side of the SPC. CM equivalent circuit with filter is shown in Fig.9. This equivalent circuit helps to derive the expression for CM attenuation $\mathrm{A}_{\mathrm{TCM}}$ with (11) to (14).

$$
\begin{gathered}
A_{T C M}=\frac{\frac{R_{\text {loadCM }} Z_{s C M}}{R_{\text {load } C M}+Z_{s C M}} I_{s C M}}{\frac{Z_{s C M}}{R_{\text {loadCM }}+Z_{s C M}+Z_{f C M}} I_{S C M} R_{\text {loadCM }}} \\
A_{T C M}=1+\frac{Z_{f C M}}{R_{\text {loadCM }}+Z_{s C M}} \\
\left|R_{\text {loadCM }}+Z_{s C M}\right|=\frac{\left|Z_{f C M}\right|}{\left|A_{T C M}-1\right|}
\end{gathered}
$$

For $\mathrm{A}_{\mathrm{TCM}}>>1$,

$$
\left|R_{\text {load } C M}+Z_{S C M}\right|=\frac{\left|Z_{f C M}\right|}{\left|A_{T C M}\right|}
$$

For example, under full load the CM noise spike of the SPC at $1 \mathrm{MHz}$ is $55 \mathrm{~dB}$. After testing $\mathrm{CM}$ inductor is added, such that the attenuation is greater than one. If $\mathrm{CM}$ noise at $1 \mathrm{MHz}$ is $35 \mathrm{~dB}$, then:

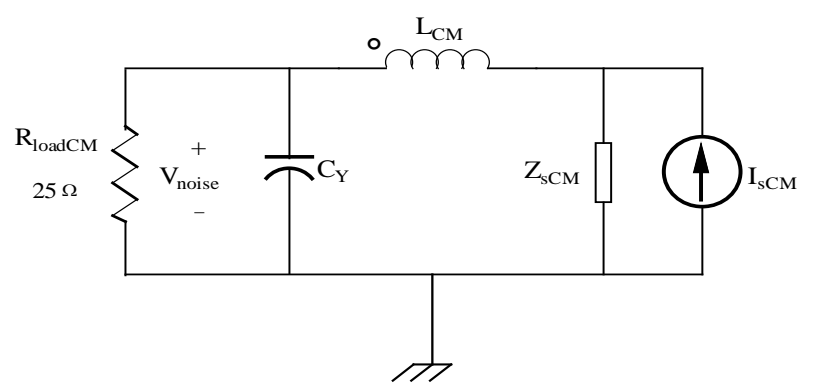

Fig. 11. CM noise equivalent circuit with the $\mathrm{CM}$ inductor at the input of the SPC.

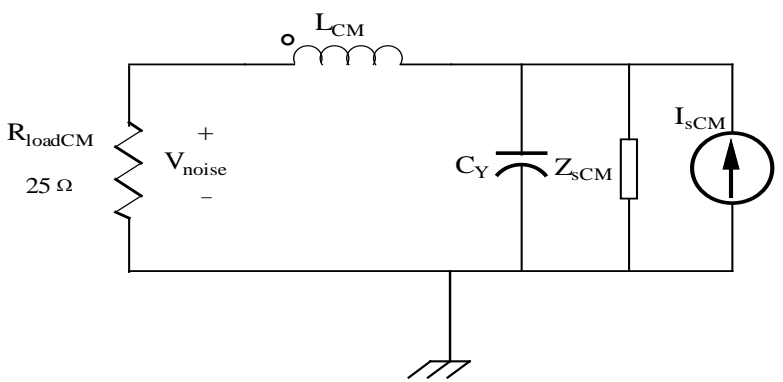

Fig. 12. CM noise equivalent circuit with the Y capacitor at the input of the SPC.

$$
\left|A_{T C M}\right|=10^{\frac{55-35}{20}}=10
$$

The impedance of the CM inductor is $\mathrm{Z}_{\mathrm{fCM}}$. $\mathrm{R}_{\text {loadCM }}$ is the equivalent resistance of the LISN. From the test results, it is noted that the CM noise is the dominant factor. Calculations are made at different frequency points using measurements of the noise voltage $\mathrm{V}_{\text {noisecm }}$ along with (10) and (15) - (18).

1) When $A_{\text {TCM }} \geq 10$ :

$$
\begin{aligned}
& \left|Z_{S C M}\right|_{\text {Max }} \approx\left|R_{\text {load } C M}+\frac{\left|Z_{f C M}\right|}{\left|A_{T C M}\right|}\right| \\
& \left|Z_{S C M}\right|_{\text {Min }} \approx\left|R_{\text {loadCM }}-\frac{\left|Z_{f C M}\right|}{\left|A_{T C M}\right|}\right|
\end{aligned}
$$

2) When $A_{\text {TCM }}<10$ :

$$
\begin{aligned}
& \left|Z_{S C M}\right|_{\text {Max }} \approx\left|R_{\text {load } C M}+\frac{\left|Z_{f C M}\right|}{\left|A_{T C M}-1\right|}\right| \\
& \left|Z_{S C M}\right|_{\text {Min }} \approx\left|R_{\text {loadCM }}-\frac{\left|Z_{f C M}\right|}{\left|A_{T C M}+1\right|}\right|
\end{aligned}
$$

In order to measure the maximum and minimum possible value of DM noise source impedance, a test capacitor is added at the input side of the SPC. DM equivalent circuit with filter is shown in Fig.10. This equivalent circuit helps to 


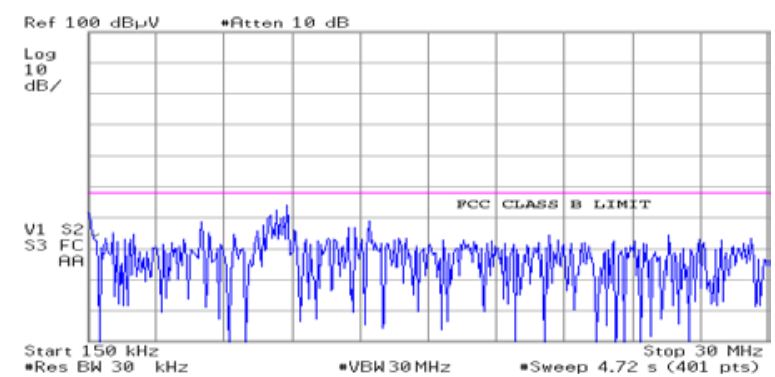

Fig.13. Noise spectrum of CM inductor at the input of the SPC.

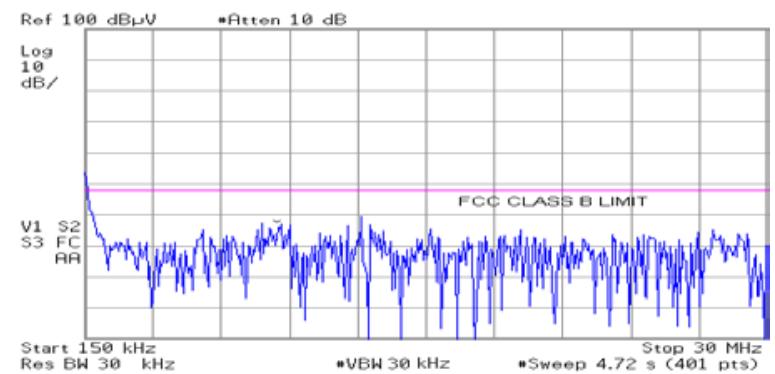

Fig. 14. Noise spectrum of Y capacitor at the input of the SPC.

derive the expression for $\mathrm{DM}$ attenuation $\mathrm{A}_{\mathrm{TDM}}$ along with (10) and (19) to (22). The maximum possible value and minimum possible value of the DM noise impedance from 0.15 to $2.5 \mathrm{MHz}$ are calculated at different frequency points using measurements of the noise voltage $V_{\text {noiseDM }} \cdot Z_{s D M}$ is the $\mathrm{DM}$ noise impedance of the SPC and $\mathrm{Z}_{\mathrm{fDM}}$ is the $\mathrm{X}$ capacitor’s impedance.

$$
\begin{aligned}
& \left|A_{T D M}\right|=\left|1+\frac{R_{\text {loadDM }} Z_{s D M}}{\left(R_{\text {loadDM }}+Z_{s D M}\right) Z_{f D M}}\right| \\
& \left|A_{T D M}\right| \approx\left|1+\frac{Z_{s D M}}{Z_{f D M}}\right| \\
& \left|Z_{S D M}\right|_{\text {Min }}=\left|Z_{f D M}\right| A_{T D M}|-1| \\
& \left|Z_{S D M}\right|_{\text {Max }}=\left|Z_{f D M}\right| A_{T D M}|+1|
\end{aligned}
$$

\section{A. CM Filter Design}

In order to design CM filter, the following must be known: 1) the attenuation required to make the CM noise spectrum pass the EMI standards at the frequencies of interest and 2) the worst case, maximum or minimum noise impedance, for the frequency range of interest. For the CM part of the EMI filter, two basic topologies are selected. In the first topology, the CM inductor faces the input of the SPC, as illustrated in Fig.11. In the second topology, the Y capacitor faces the input of the SPC, as illustrated in Fig.12.

Using the equivalent circuits of Fig.11 and Fig.12 the CM noise voltage across $R_{\text {loadCM }}$ is calculated when the filter is added. Two topologies are tested. For the test, the CM inductance used is $100 \mu \mathrm{H}$ and the Y capacitor used is $330 \mathrm{nF}$. Fig.13 shows the noise spectrum of the CM inductor at the input of the SPC. The noise spectrum of the Y capacitor at the

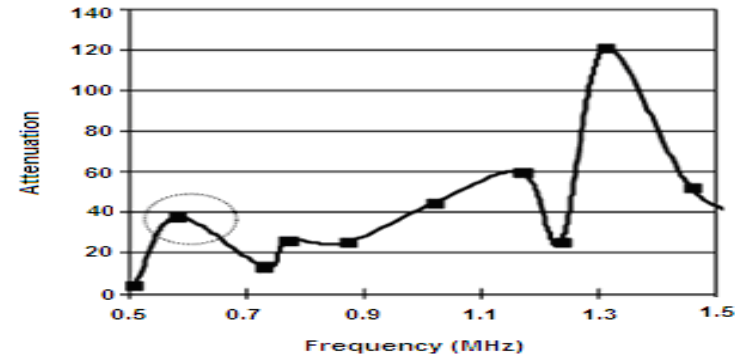

Fig.15. Attenuation required for the CM Noise Spectrum.

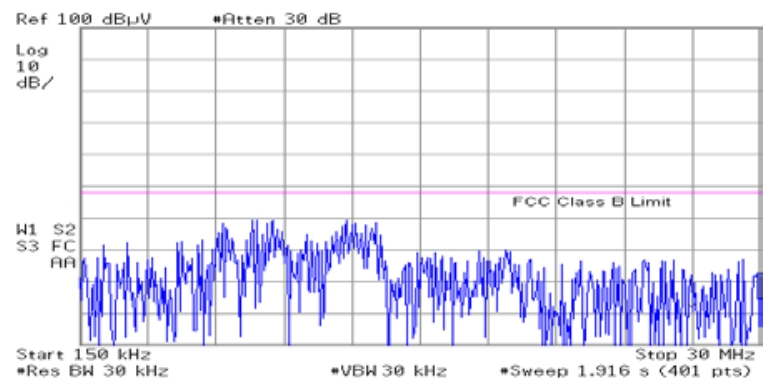

Fig. 16. Test results of the CM noise spectrum after the CM filter designed based on maximum noise impedance is added.

input of the SPC is shown in Fig. 14.

From the results, it is concluded that the topology of Fig. 11 should be used because it provided more attenuation at high frequencies than the topology shown in Fig. 12. These results also emphasize the fact that noise impedance has a significant effect on the performance of the EMI filter. In order to determine the lowest corner frequency required for the CM filter, (23) is used as follows:

$$
f_{C}=\frac{F_{0}}{\sqrt{A_{\text {TCMreq }}}}
$$

$A_{\text {TCMreq }}$ is the required attenuation at frequency $F_{0}$, and $f_{c}$ is the corner frequency of the $\mathrm{CM}$ filter. The $\mathrm{CM}$ attenuation required for the SPC to pass the FCC 15 Class B standards with a 3-dB margin is plotted over the frequency range from 0.5-1.5 MHz, as shown in Fig.15. Equation (23) is evaluated at each point for the data presented in Fig.15. The lowest calculated filter corner frequency is $\mathrm{f}_{\mathrm{c}}=94 \mathrm{kHz}$, using $\mathrm{F}_{0}=$ $583 \mathrm{kHz}$ at $\mathrm{A}_{\text {TCMreq }}=38$. To select the value of the $\mathrm{CM}$ inductor, the maximum noise impedance is used because it provides the least attenuation for the filter in Fig.11, where the inductor faces the input of the SPC. When the phase angle of the noise impedance is neglected, and to guarantee that the CM noise impedance has little effect on the performance of the EMI filter, the impedance of the CM inductor at the frequency of interest must be much larger than the maximum magnitude of the CM noise impedance.

In fact, to make sure that the desired attenuation is achieved, the CM inductor's impedance must be at least double the maximum magnitude of the CM noise impedance. 


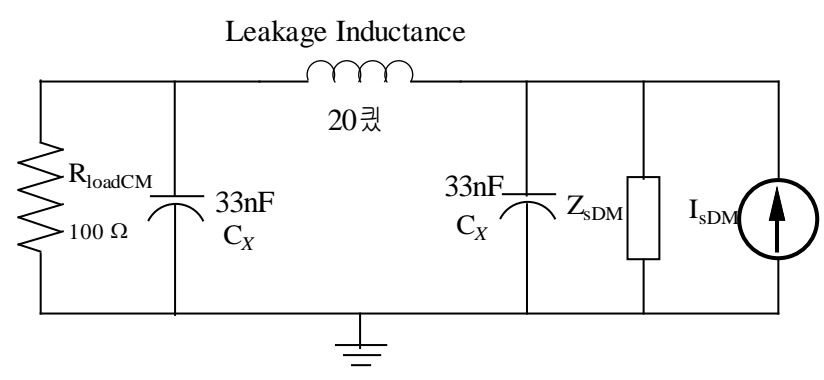

Fig. 17. DM noise equivalent circuit including the л DM filter topology.

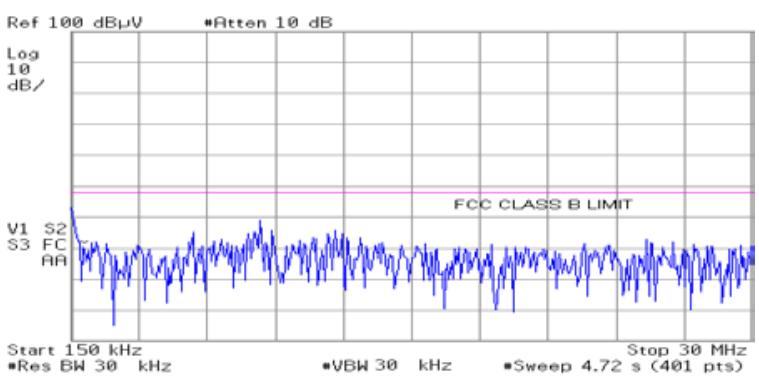

Fig. 18. Test results for DM noise spectrum after the PI filter is added.

Therefore, the CM inductor's impedance at $\mathrm{F}_{0}$ must be larger than $2 \times 20 \Omega \Omega=410 \Omega$, which corresponds to an inductance greater than $2 \times 55.9 \mu \mathrm{H}=111.8 \mu \mathrm{H}$. The inductance chosen is any reasonable value above $111.8 \mu \mathrm{H}$. In order to leave some margin, a $200 \mu \mathrm{H}$ inductance is selected. The impedance of the $\mathrm{CM}$ inductor at $583 \mathrm{kHz}$ is $73 \Omega$ which is approximately three times the maximum magnitude of the CM noise impedance. Ignoring the phase angle of the CM noise impedance (i.e., considering the CM noise impedance as purely resistive), the attenuation of the topology in Fig.11, is expressed by (24), where $V_{1}$ and $V_{2}$ are given by (25) and (26), respectively. The parameters $Z_{1}$ and $Z_{2}$ are given by (27) and (28), respectively.

$$
\begin{aligned}
& \left|A_{T C M}\right|=\left|\frac{V_{2}}{V_{1}}\right| \\
& V_{1}=\frac{Z_{1} Z_{2}}{\left(Z_{L C M}+Z_{1}\right)} I_{s C M} \\
& V_{2}=\frac{R_{\text {load }} Z_{s C M}}{R_{\text {load }}+Z_{S C M}} I_{s C M} \\
& Z_{1}=\frac{R_{\text {load }} Z_{C Y}}{R_{\text {load }}+Z_{C Y}} \\
& Z_{2}=\left(Z_{L C M}+Z_{1}\right)\left(\frac{Z_{S C M}}{Z_{S C M}+Z_{1}+Z_{L C M}}\right)
\end{aligned}
$$

$\mathrm{Z}_{\mathrm{LCM}}$ is the impedance of the CM inductor. $\mathrm{Z}_{\mathrm{CY}}$ is the impedance of the $\mathrm{Y}$ capacitor. $\mathrm{V}_{2}$ is the voltage across the LISN equivalent resistor shown in Fig. 11 and it is the noise voltage across the LISN resistor without the CM filter. The final step in the filter design is to select the capacitance. A

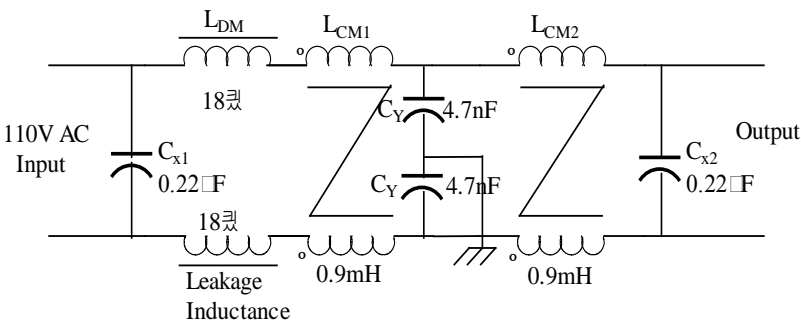

Fig. 19. Complete EMI filter containing the DM and CM filters for the ac-dc SPC.

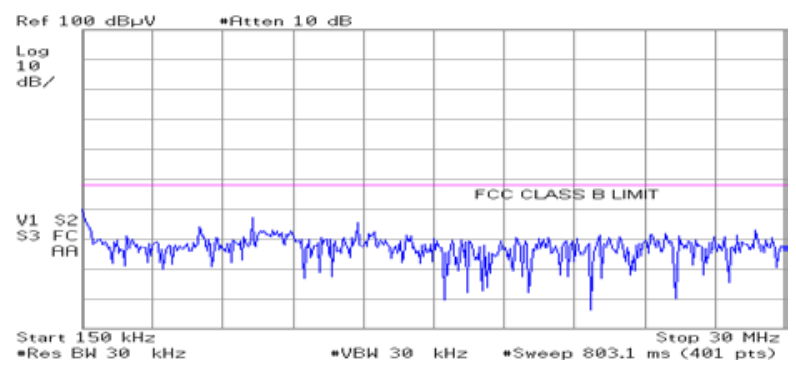

Fig. 20. Test results of the total noise spectrum after the EMI filter is added to the input of the ac-dc SPC.

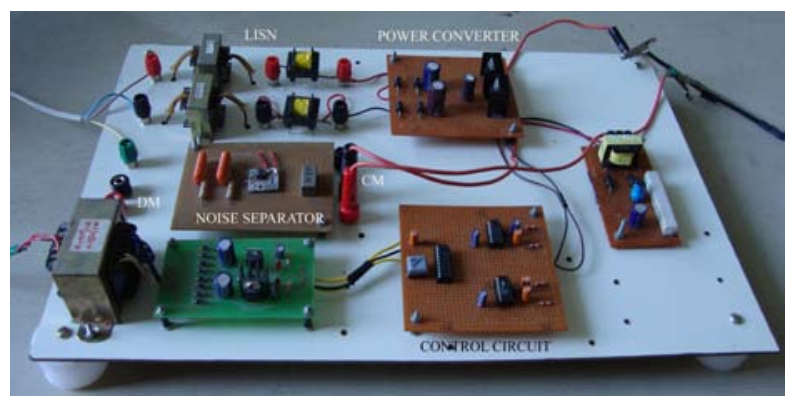

Fig. 21. Photograph of the measurement arrangement.

capacitance of $136 \mathrm{nF}$ is selected. This is achieved by using two $\mathrm{C}_{\mathrm{Y}}$ capacitors, $68 \mathrm{nF}$ each, in parallel. Using this capacitance and (24), the calculated attenuation of the filter at $583 \mathrm{kHz}$ is 41 , which is greater than the required attenuation of 38. Fig. 16 shows the experimental results of the CM spectrum after apply the CM filter designed using the maximum noise impedance. It is noted that the SPC with the CM filter passed the FCC 15 Class B requirements over the required frequency range and that the attenuation achieved is greater than the 3-dB margin over most of the frequency range.

If the capacitance is selected based on an ideal LC filter design, the calculated capacitance required will be $14.3 \mathrm{nF}$ using (29). The closest available capacitance is $16.4 \mathrm{nF}$ (2 $\mathrm{x}$ $8.2 \mathrm{nF}$ in parallel)

$$
f_{C}=\frac{F_{0}}{2 \pi \sqrt{L_{C M} C_{Y}}}
$$

Then, by using (24), with the following parameters $\mathrm{C}_{\mathrm{Y}}=$ $16.4 \mathrm{nF}, \mathrm{R}_{\text {loadCM }}=25 \Omega, \mathrm{L}_{\mathrm{CM}}=200 \mu \mathrm{H}$, and $\mathrm{Z}_{\mathrm{SCM}}=205 \Omega$, the 
calculated attenuation is 5.9 at $583 \mathrm{kHz}$. This is less than the required attenuation, $\mathrm{A}_{\text {TCMreq }}=38$. It is obvious that this filter does not meet the FCC Class $B$ requirements at approximately $560 \mathrm{kHz}$. From the above analysis, it is clear that neglecting the noise impedance can lead a designer to design a filter that will not meet the FCC requirements. By neglecting the noise impedance, the selected $\mathrm{Y}$ capacitance is $16.4 \mathrm{nF}$. However, when the noise impedance is taken into account, the selected capacitance of $136 \mathrm{nF}$ is nearly ten times larger. The experiment results also verify this conclusion. From the above analysis and experimental results it is again demonstrated that the noise impedance has a significant effect on the performance of the EMI filter.

\section{B. DM Filter Design}

The DM filter design method is similar to that of the CM filter. In order to design DM filter, the following must be known: 1) the attenuation required for making the DM noise spectrum pass the EMI standards at the frequencies of interest and 2) the maximum and minimum possible values of the noise impedance for the frequency range of interest. А л topology is selected to suppress the DM noise, because this topology provides better performance than other simple topologies such as the LC topology. This topology, along with a noise source, its impedance and $\mathrm{R}_{\text {loadDM }}$, is displayed in Fig. 17. Since an $\mathrm{X}$ capacitor is used at the input of the SPC, the minimum DM noise impedance is used for the filter design. The attenuation of this topology is expressed using (30), where $Z_{C l}$ and $Z_{C s}$ are given by (31) and (32), respectively. In (31) and (32) $Z_{C X}$ is the impedance of the $X$ capacitor, and in (30) $\mathrm{Z}_{\mathrm{LDM}}$ is the impedance of the differential mode inductance. In (30) and (31) $\mathrm{R}_{\text {loadDM }}$ is the LISN equivalent load resistance of $100 \Omega$.

$$
\begin{gathered}
\left|A_{T D M}\right|=\left|\frac{R_{\text {load } D M} Z_{s D M}\left(Z_{C 1}+Z_{L D M}+Z_{C s}\right)}{\left(R_{\text {load } C M}+Z_{s D M}\right) Z_{C s} Z_{C 1}}\right| \\
Z_{C 1}=\frac{R_{\text {load } C M} Z_{C X}}{R_{\text {load } C M}+Z_{C X}} \\
Z_{C s}=\frac{Z_{C X} Z_{s D M}}{Z_{C X}+Z_{s D M}}
\end{gathered}
$$

Using the experimental results, the lowest corner frequency required is at $\mathrm{F}_{0}=583 \mathrm{kHz}$. At this frequency, the attenuation needed is 4.5 . It should be noted that the leakage inductance of the CM inductor is used as the DM inductance. As a result, only one inductor is required to meet the attenuation requirements for CM and DM noise. Using (30), where $\mathrm{L}_{\mathrm{DM}}$ $=20 \mu \mathrm{H}, \mathrm{Z}_{\mathrm{sDMmin}}=0.06 \Omega$, and $\mathrm{C}_{\mathrm{X}}=33 \mathrm{nF}$, the calculated attenuation $A_{\mathrm{TDM}}$ is 7.87 at $\mathrm{F}_{0}=583 \mathrm{kHz}$, which is greater than the required attenuation of 4.5 . The test results of the DM noise spectrum after the DM filter is added are shown in Fig. 18. It is clear that the DM filter exceeded the FCC 15
TABLE I

COMPARISON OF HARDWARE WAVEFORM RESUlTS

\begin{tabular}{|c|c|c|c|c|c|c|}
\hline \multirow{2}{*}{$\begin{array}{c}\text { Frequency } \\
\text { in MHz }\end{array}$} & \multicolumn{3}{|c|}{$\begin{array}{c}\text { Without Filter } \\
(\mathrm{dB} \mu \mathrm{V})\end{array}$} & \multicolumn{3}{c|}{$\begin{array}{c}\text { With Filter } \\
(\mathrm{dB} \mu \mathrm{V})\end{array}$} \\
\cline { 2 - 7 } & Total & $\mathrm{CM}$ & $\mathrm{DM}$ & $\mathrm{CM}$ & $\mathrm{DM}$ & EMI \\
\hline 10 & 83 & 79 & 74 & 35 & 32 & 28 \\
\hline 20 & 80 & 76 & 73 & 25 & 24 & 23 \\
\hline 30 & 78 & 74 & 71 & 24 & 23 & 22 \\
\hline
\end{tabular}

Class B specifications over the entire frequency range by more than $3 \mathrm{~dB}$.

\section{Complete EMI Filter Design}

Finally, the CM and DM filters are combined. The resulting EMI filter is illustrated in Fig. 19. A 200- $\mu \mathrm{F}$ electrolytic capacitor is added to the output of the filter to ensure that the EMI filter will not destabilize the feedback loop of the SPC [18]. Since the resonant frequency of this electrolytic capacitor is relatively low, it does not affect the filter design. The test results for the filter are shown in Fig.20. From the test results, it is noted that the filter allows the SPC to pass the FCC 15 Class B requirements for conducted EMI. The proposed method is used to design an EMI filter for an ac-dc power converter. Experimental results are presented for an isolated ac-dc half-bridge converter with a nominal input voltage of $220 \mathrm{~V} / 50 \mathrm{~Hz}$, and an output voltage of $12 \mathrm{~V}_{\mathrm{DC}}$ under a full load current of $5 \mathrm{~A}$. After measuring the DM and $\mathrm{CM}$ noise spectrum and calculating the noise impedance, the filter topology is selected [19]. A T topology is selected for CM filter because the worst case CM noise impedance is calculated to be $1.9 \mathrm{k} \Omega$. The attenuation required is 57 at 0.6 $\mathrm{MHz}$.

А л topology is selected to suppress the DM noise, since this topology provides higher attenuation than other simple topologies such as the LC topology. The worst case DM noise impedance is $3 \Omega$. The required attenuation is 20 at 0.15 $\mathrm{MHz}$. It is clear that the filter exceeded the FCC 15 Class B specifications across the entire frequency range by more than $3 \mathrm{~dB}$ [20]. Table.1 shows comparison of the hardware results for various frequency ranges using filters. Fig.16 shows photograph of the measurement arrangement.

The method presented is not limited to single power converter. It is used to design EMI filters for paralleled switching power converter modules. The paralleled power modules are simplified into one equivalent power module. Therefore, the method presented is used to design the EMI filter for a power converter consisting of multiple power converters. An example of an EMI filter for a Half-bridge isolated ac-dc converter is designed to illustrate the proposed method. As expected, the Half-bridge isolated ac-dc converter 


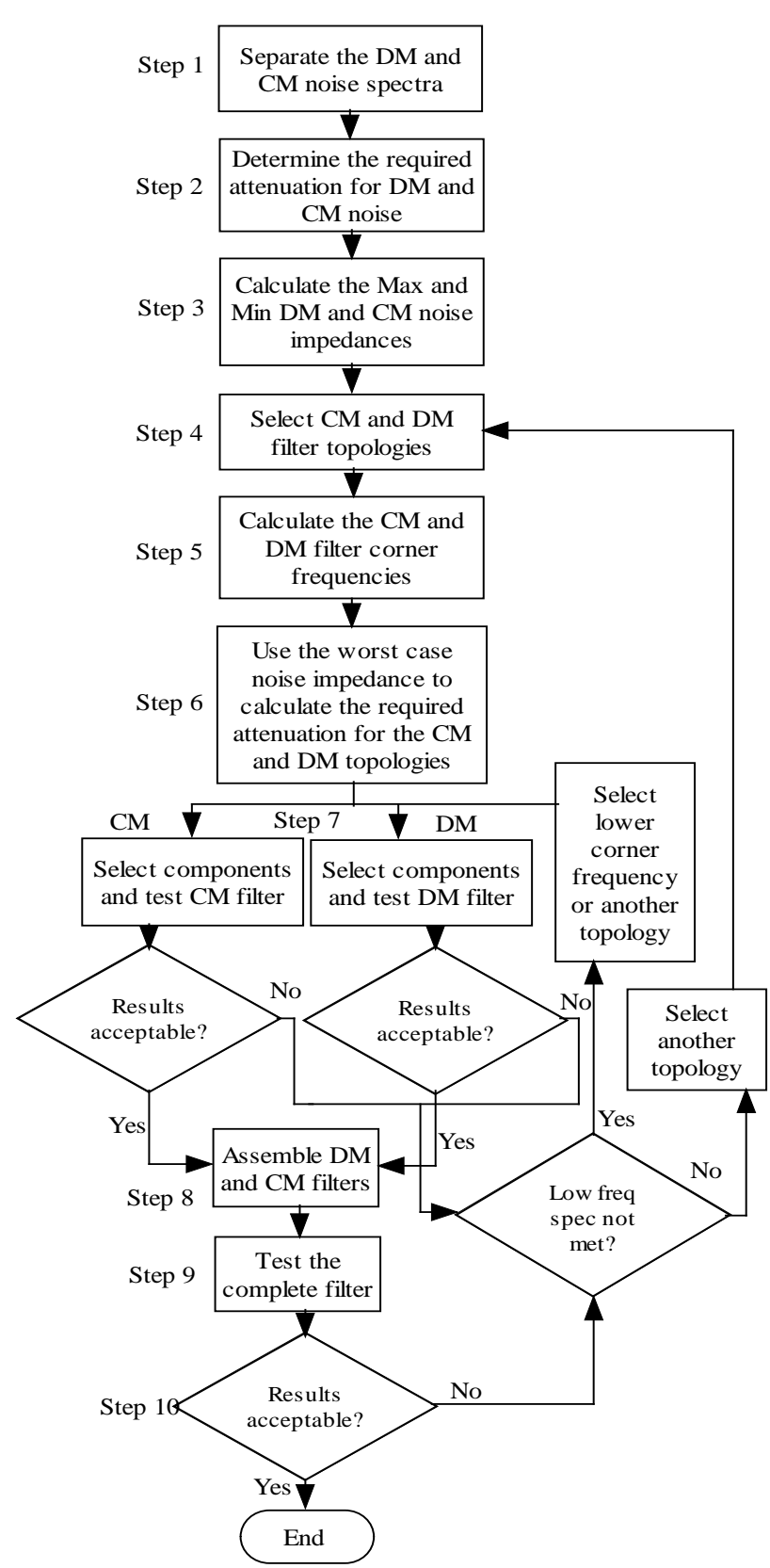

Fig. 22. Flowchart of the filter design method.

with the designed filter meets the requirements set in the standard. Fig 22 shows flowchart of the proposed filter design method.

\section{CONCLUSIONS}

The proposed method is based on measuring the noise spectrum and using the data to calculate the maximum possible and minimum possible values of the noise impedance, and using then in the EMI filter design. This approach considers the filter topology only but does not mention the relationship between the noise source impedance and noise load impedance, which determines the EMI filter performance significantly. Various filters like LC filter, $\pi$ filter and complete EMI filters are analyzed. From Table I, it is seen that the waveforms with filters are below the FCC class B level. The analysis and results proposed here are used to provide a guideline for improving the effectiveness of future filtering schemes in switching power converters. The estimation of parameters for the model can be done by measurement or analytical calculations based on the geometry of the circuit. Based on the model and analysis proposed in this paper, future works will focus on parameter extraction and accurate EMI prediction.

Experimental Results have shown that the proposed method is very effective and accurate in identifying and capturing EMI features in switching power converters. The method presented is not only limited to half-bridge converters. It can be applied to many different converter topologies, such as buck, boost and flyback converters with single-phase diode bridge front-end. This model contains the salient features of conducted EMI. It is convenient to use and gives adequate prediction of the EMI behavior in switching converters.

\section{REFERENCES}

[1] L. Ran, "Conducted electromagnetic emissions in induction motor drive systems Part I: Time domain analysis and identification of dominant modes," IEEE Transactions on Power Electronics, Vol. 13, No. 4, pp. 757-767, July 1998.

[2] L. Ran, "Conducted electromagnetic emissions in induction motor drive systems Part II: Frequency domain models," IEEE Transactions on Power Electronics, Vol. 13, No. 4, pp. 768-776, July 1998.

[3] M.Jin, M.Weiming, P.Qijun, K.Jun, Z.Lei and Z.Zhihua, "Identification of essential coupling path models for conducted EMI prediction in switching power converters," IEEE Transactions on Power Electronics, Vol. 21, No. 6, pp. 1795-1803, Nov. 2006.

[4] F. Y. Shih et al., "A procedure for designing EMI filters for AC line applications," IEEE Transactions on Power Electronics, Vol. 11, No. 1, pp. 170-181, Jan. 1996.

[5] T. Guo, D. Y. Chen, and F. C. Lee, "Separation of the common-mode and differential-mode-conducted EMI noise,” IEEE Transactions on Power Electronics, Vol. 11, No. 3, pp. 480-488, May 1996.

[6] Y. Zhao, K.Y. See, "Fundamental of Electromagnetic Compatibility and Application,” Beijing, China Machine Press, pp. 165-174, 2007.

[7] J. R. Regue, M. Ribo, "Common and differential mode characterization of EMI power-line filters from S-parameters measurements," IEEE International Symposium on Electromagnetic Compatibility, Vol. 2, pp. 610-615, 2004.

[8] F. M. Tesche, "EMC Analysis Methods and Computational Models,” Wiley, New York, 1997.

[9] L. Ran, J. C. Clare, K. J. Bradley, and C. Christopoulos, "Measurement of conducted electromagnetic emissions in PWM motor drive systems without the need for an LISN," IEEE Transactions on Electromagnetic Compatibility, Vol.41, No. 1, pp. 50-55, Mar. 1999.

[10] D. Zhang et al., "Measurement of noise source impedance of off-line converters," IEEE Transactions on Power Electronics, Vol. 15, No. 5, pp. 820-825, Sep. 2000. 
[11] C. R. Paul, "Introduction to Electromagnetic Compatibility," Wiley, New York, 1992.

[12] R. Vimala, K. Baskaran, K. R. Aravind Britto, "Characterization of Conducted EMI Generated by Switched Power Converters," International Journal of Recent Trends in Engineering, Vol.1, No. 3, pp. 305-307, May 2009.

[13] R. W. Erickson, "Fundamentals of Power Electronics," Springer-Verlag, NewYork, 1997.

[14] B. Choi and B. Cho, "Intermediate line filter design to meet both impedance compatibility and EMI specifications," IEEE Transactions on Power Electronics, Vol. 10, No. 5, pp.583-588, Sep. 1995.

[15] B. Choi, D. Kim, D. Lee, S. Choi, and J. Sun, "Analysis of input filter interactions in switching power converters," IEEE Transactions on Power Electronics, Vol. 22, No. 2, pp. 452-460, Mar. 2007.

[16] M. Nave, "Power Line Filter Design for Switched Mode Power Supplies," Van Nostrand, New York, 1991.

[17] R.Vimala, K.Baskaran, K.R.Aravind Britto, "Determination of Maximum and Minimum Possible CM and DM Noise Impedances," International journal of Electronics \& Telecommunication and Instrumentation Engineering, Vol. 1, No. 1, pp. 24-31, March 2010.

[18] Input System Instability Application Note PQ-00-05-1 Rev. 01, [Online] Available:http://www.synqor.com/support/3_ 1_app_notes. htm, 2004.

[19] R.Vimala, K.Baskaran, N.Devarajan, "Modeling and Filter Analysis of Differential Mode EMI for Switching Power Converters," European Journal of Scientific Research, Vol. 52, No.4, pp.553-568, April 2011.

[20] R.Vimala, K.Baskaran, K.R.Aravind Britto, "Modeling of Conducted EMI in Switching Power Converters using Equivalent Circuit Model," International Journal of Electrical Engineering, Vol. 4, No. 1, pp. 59-74, April 2011.

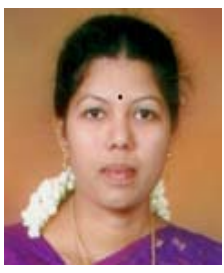

R. Vimala received her B.E. degree in Electrical and Electronics Engineering from Periyar University, Government College of Engineering, Salem, Tamil Nadu, India, in 2003, and her M.E. degree in Power Electronics and Drives from Anna University, Government College of Technology, Coimbatore, India, in 2006. She is currently working toward her Ph.D. degree in Electrical Engineering. She is a member of Indian Society for Technical Education (ISTE). She is now an Associate Professor in the Department of Electrical and Electronics Engineering, PSNA College of Engineering and Technology, Dindigul, India. Her research interests include electromagnetic compatibility of power conversion, developing models for EMI characteristics prediction, EMI reduction techniques, and computer-aided circuit simulation.

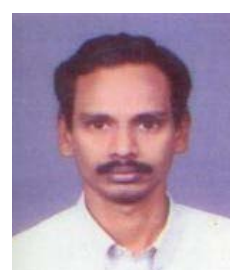

K. Baskaran received his B.E. in Electrical and Electronics Engineering from Annamalai University, India, in 1989, his M.E. in Computer Science Engineering from Bharathiar University, India, in 2002, and his Ph.D. from Anna University - Chennai, India, in 2006. He is a member of IEEE and Indian Society for Technical Education (ISTE). He is now an Associate Professor in the Department of Computer Science and Engineering, Government College of Technology, Coimbatore, India. His research interests include Adhoc networks, network security, electrical system control, etc.

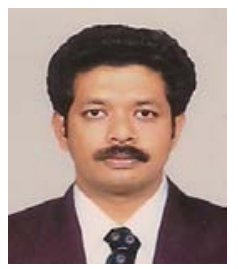

K. R. Aravind Britto received his B.E. in Electrical and Electronics Engineering from Madurai Kamaraj University, India, in 2003, and his M.E. in Applied Electronics from Anna University, India, in 2005. He is currently working toward his Ph.D. degree in Electrical Engineering. He is a member of Indian Society for Technical Education (ISTE). He is now an Associate Professor in the Department of Electronics and Communication Engineering, RVS College of Engineering and Technology, Dindigul, India. His research interests include electromagnetic compatibility, EMI characteristics prediction, EMI reduction techniques, etc. 